\title{
Complex degree of coherency inherent to laser images of blood plasma polycrystalline networks
}

\author{
Yu.A. Ushenko, V.V. Istratiy, A.V. Dubolazov, A.P. Angelsky \\ * Chernivtsi National University, Department for Correlation Optics, \\ 2, vul. Kotsyubyns'kogo, 58012 Chernivtsi, Ukraine; yuriyu@gmail.com \\ ** Chernivtsi National University, Department for Optics and Spectroscopy, \\ 2, vul. Kotsyubyns'kogo, 58012 Chernivtsi, Ukraine
}

\begin{abstract}
Given in this paper are theoretical basics for correlation-phase analysis of laser images inherent to human blood plasma. Also presented are comparative results of measurements aimed at coordinate distributions of the module of complex degree of coherency (CDC) and complex degree of mutual polarization (CDMP) of laser images describing blood plasma of a healthy person as well as of a patient with prostate cancer of the first stage. The authors investigated both values and ranges of changing the statistical (moments of the first to fourth orders), correlation (coefficients of the GrammCharlie expansion for autocorrelation functions) and fractal (slopes and dispersion of extremes for logarithmic dependences of power spectra) parameters for coordinate distributions CDC and CDMP. Determined are objective criteria for diagnostics of cancer changes in blood plasma of a patient with cancer.
\end{abstract}

Keywords: laser, polarization, correlation, blood plasma, birefringence, crystal, statistical moment, fractal.

Manuscript received 23.07.10; accepted for publication 02.12.10; published online 30.12.10.

\section{Introduction}

Among the diversity of directions for optical diagnostics of biological objects, polarization methods are a most popular [1 - 39]. The latter are based on such fundamental conceptions as "matrix of coherency" and "degree of polarization" that describe the field of scattered radiation $[1,2]$. These parameters characterize correlation similarity of orthogonal components $E_{x}(r), E_{y}(r)$ of electromagnetic wave amplitudes in separate points of optical field with coordinates $(r)$ [3]. In this sense, these analytical approaches will be considered as the "one-point" ones. They serve as a base for development of methods providing polarization mapping biological tissues (BT) and diagnostics of their pathological changes in structure (e.g., cancer of women's reproductive organs) [13-15].

Wide application of modern laser technique in investigations of BT structures stimulates development of essentially new approaches to analysis and description of polarization-inhomogeneous fields of scattered coherent radiation. One-point methods got their development in a more general "two-point" approach based on the analysis aimed at the degree of coherency between polarization states of adjacent $\left(r_{1}, r_{1}+\Delta r\right)$ points in the field of scattered radiation [4-11]. From the quantitative viewpoint, this correlation may be characterized with the value of module for complex degree of mutual polarization (CDMP) $V\left(r_{1}, r_{1}+\Delta r\right)$ [12]. In the works $\{13,29]$, based on CDMP developed was the method of polarization-correlation mapping (PCM) for an optically-anisotropic BT structure. It was based on the analysis of coordinate distributions $V\left(r_{1}, r_{1}+\Delta r\right)$. As a result, the authors realized not only diagnostics, but also differentiation of severity inherent to oncological changes (precancer - cancer of the first to fourth stages) in tissues of woman's reproductive sphere [18].

On the other hand, there is a wide circle of weakly anisotropic biological liquids (BL) taken from a human organism, which are considerably more accessible as compared with BT samples that need a traumatic biopsy operation. From the physical viewpoint, BL are matter with a weak polarization modulation of laser radiation 
$[33,35,37]$. Thereof, the task of using phase information contained in the field of radiation scattered by BL is topical.

Our work is aimed at development and testing the two-point correlation-phase method in investigations of blood plasma in order to provide early diagnostics of oncological changes in human organs (e.g., prostate).

\section{Model conceptions}

As a basis for the analysis of phase structure inherent to the field of laser radiation transformed by blood plasma, we used the following model $[13,17,18,21,22,25,28$, $32,33,35]$ :

- blood plasma is considered as a two-component isotropic-anisotropic structure;

- optically isotropic component is the fraction consisting of optically single-axis birefringent crystals of albumin and globulin amino acids;

- phase properties of these biological crystals are characterized with the Jones matrix

$\{D\}=\left\|\begin{array}{ll}d_{11} & d_{12} \\ d_{21} & d_{22}\end{array}\right\|$,

where

$$
d_{i k}(r, \rho, \delta)=\left\{\begin{array}{l}
d_{11}=\cos ^{2} \rho(r)+\sin ^{2} \rho(r) \exp (-i \delta(r)) ; \\
d_{12}=d_{21}=\cos \rho(r) \sin \rho(r)(1-\exp (-i \delta(r))) ; \\
d_{22}=\sin ^{2} \rho(r)+\cos ^{2} \rho(r) \exp (-i \delta(r)) .
\end{array}\right.
$$

Here, $\rho$ is the direction of the optical axis; $\delta=2 \pi / \lambda \Delta$ nd - phase shift between orthogonal components of the amplitude; $\lambda$ - wavelength; $d-$ geometric distance; $\Delta n$ - birefringency coefficient.

\section{Brief theory of the correlation-phase method}

As a basis for "two-point" correlation-phase method providing investigation of blood plasma, we used the conception of CDC for points of its laser image. The parameter $\mu\left(r_{1}, r_{2}\right)$ below characterizes correlation between orthogonal components $\left(E_{x}, E_{y}\right)$ of the amplitude of laser field in two points with coordinates $r_{1}$ and $r_{2}$

$\mu\left(r_{1}, r_{2}\right)=\left[\frac{\operatorname{Tr}\left(W^{\diamond}\left(r_{1}, r_{2}\right) W\left(r_{1}, r_{2}\right)\right)}{\operatorname{Tr} W\left(r_{1}, r_{1}\right) \cdot \operatorname{Tr} W\left(r_{2}, r_{2}\right)}\right]$.

Here $W\left(r_{1}, r_{2}\right)$ is the transverse spectral density matrix of the following form

$$
W\left(r_{1}, r_{2}\right)=\left[\begin{array}{ll}
E_{x}^{*}\left(r_{1}\right) E_{x}\left(r_{2}\right) & E_{x}^{*}\left(r_{1}\right) E_{y}\left(r_{2}\right) \\
E_{y}^{*}\left(r_{1}\right) E_{x}\left(r_{2}\right) & E_{y}^{*}\left(r_{1}\right) E_{y}\left(r_{2}\right)
\end{array}\right],
$$

where $W^{\diamond}\left(r_{1}, r_{2}\right)$ is the Hermitian conjugate matrix to $W\left(r_{1}, r_{2}\right) ; \operatorname{Tr}$ - spur of the matrix.

Let us write the expression (3) for laser field transformed by a biological crystal (relations (1) and (2)) in two its arbitrary points. In this case, the transverse spectral matrix (relation (4)) for the density of this field takes a look

$W_{\text {out }}\left(r_{1}, \mathrm{r}_{2}\right)=D^{\diamond}\left(r_{1}\right) \cdot W_{\text {in }}\left(r_{1}, r_{2}\right) \cdot D\left(r_{2}\right)$.

Here $D\left(r_{1}\right)$ and $D\left(r_{2}\right)$ are Jones matrixes for the biological crystal in the points $r_{1}$ and $r_{2} ; W_{i n}\left(x_{1}, x_{2}\right)$ transverse spectral density matrix for the probing laser beam

$W_{i n}\left(r_{1}, r_{2}\right)=\left[\begin{array}{ll}E_{x}^{*}\left(r_{1}\right) E_{x}\left(r_{2}\right) & E_{x}^{*}\left(r_{1}\right) E_{y}\left(r_{2}\right) \\ E_{y}^{*}\left(r_{1}\right) E_{x}\left(r_{2}\right) & E_{y}^{*}\left(r_{1}\right) E_{y}\left(r_{2}\right)\end{array}\right]$.

With account of the expressions (1) to (6), the expression $\mu\left(r_{1}, r_{2}\right)$ takes the following look

$\mu\left(r_{1}, r_{2}\right)=\sqrt{\frac{1}{(a+i b)\left(\cos ^{2} \Delta \rho_{12}+\sin ^{2} \Delta \rho_{12} \exp \left(-i \cdot 2 \Delta \delta_{12}\right)\right)}}$.

Here, $\Delta \rho_{12}=\rho\left(r_{1}\right)-\rho\left(r_{2}\right), \quad \Delta \delta_{12}=\delta\left(r_{1}\right)-\delta\left(r_{2}\right)$, and $(a+i b)$ is the coefficient of proportionality.

The expression (7) shows a simultaneous dependence of the CDC value for blood plasma image both on the orientation $(\Delta \rho)$ and phase $(\Delta \delta)$ structure of its polycrystalline network. To eliminate this ambiguity one can using laser probing beam with circular polarization. In this case, Exp. (7) is transformed into the only phase dependence

$\mu\left(r_{1}, r_{2}\right)=\sqrt{\frac{1}{\left(\exp \left(-i 2 \Delta \delta_{12}\right)+1\right)}}$.

In what follows (without losses in completeness of analysis), let us confine ourselves by taking into account the CDC module $\left|\mu\left(r_{1}, r_{2}\right)\right|$

$\left|\mu\left(r_{1}, r_{2}\right)\right|=0.5\left(1+\cos 2 \Delta \delta_{12}\right)^{-1}$.

Thus, to determine the value of CDC module we need information about the difference between phase shifts $\delta\left(r_{1}\right)-\delta\left(r_{2}\right)$ inherent to orthogonal components of amplitudes $E_{x}\left(r_{1}\right), E_{y}\left(r_{1}\right)$ and $E_{x}\left(r_{2}\right), E_{y}\left(r_{2}\right)$ in the points with coordinates $r_{1}, r_{2}$.

To obtain this information, let us consider the process of formation of the laser image $\left(E_{0} \rightarrow E(r \equiv x, y)\right)$ for the layer of blood plasma $(\{D(r)\})$ that is placed between two phase filters - 
quarter-wave plates $\left(\left\{\Phi_{1}\right\},\left\{\Phi_{2}\right\}\right)$ and polarizers $\left(\left\{P_{1}\right\}\right.$, $\left\{P_{2}\right\}$ ), transmission planes of which make $+45^{\circ}$ and $-45^{\circ}$ angles with directions of maximum velocity axes.

This optical arrangement provides two functions simultaneously:

- formation of the circular-polarized laser beam $E_{0}^{*}=\left\{\Phi_{1}\right\}\left\{P_{1}\right\} E_{0}$ that probes blood plasma;

- direct measurement of values for the phase shift $\delta$ between orthogonal components of the laser wave amplitudes $E\left(E_{x}, E_{y}\right)$ in the points with coordinates $r$.

Let us consider this phase-metric process in detail. The amplitude $E(r)$ in every point of polarizationfiltered laser image describing blood plasma can be represented with the following matrix equation

$E(r)=0.25\left\{P_{2}\right\}\left\{\Phi_{2}\right\}\{D(r)\}\left\{\Phi_{1}\right\}\left\{P_{1}\right\} E_{0}$.

Here

$\left\{\begin{array}{l}E_{0}=\left(\begin{array}{l}E_{0 x} \\ E_{0 y} \exp \left(-i \delta_{0}\right)\end{array}\right), \quad E(r)=\left(\begin{array}{l}E_{x}(r) \\ E_{y}(r) \exp (-i \delta(r))\end{array}\right), \\ \left\{P_{1}\right\}=\left\|\begin{array}{ll}1 & 1 \\ 1 & 1\end{array}\right\|, \quad\left\{P_{2}\right\}=\left\|\begin{array}{cc}1 & -1 \\ -1 & 1\end{array}\right\|, \quad\left\{\Phi_{1}\right\}=\left\|\begin{array}{ll}1 & 0 \\ 0 & i\end{array}\right\|, \quad\left\{\Phi_{2}\right\}=\left\|\begin{array}{ll}i & 0 \\ 0 & 1\end{array}\right\| .\end{array}\right.$

In a particular case of linearly-polarized laser radiation $\left(E_{0}=\left(\begin{array}{l}1 \\ 1\end{array}\right)\right.$, Eq. (10) takes a look $E(r)=0.25\left|\begin{array}{cc}1 & -1 \\ -1 & 1\end{array}\left\|\mid \begin{array}{cc}i & 0 \\ 0 & 1\end{array}\right\| \times\right.$ $\left\|\begin{array}{ll}\cos ^{2} \rho(r)+\sin ^{2} \rho(r) \exp [-i \delta(r)] & \cos \rho(r) \sin \rho(r)\{1-\exp [-i \delta(r)]\} \\ \cos \rho(r) \sin \rho(r)\{1-\exp [-i \delta(r)]\} & \sin ^{2} \rho(r)+\cos ^{2} \rho(r) \exp [-i \delta(r))\end{array}\right\| \times$

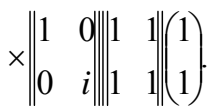

Solution of the matrix equation (12) is values of complex amplitudes $E(r)$ that are exclusively determined by the phase shift $\delta(r)$ and do not depend on the orientation of the optical axis $\rho(r)$ inherent to a biological crystal.
Thus, the intensity $I(r)$ of every point in the polarization-filtered laser image of blood plasma layer is defined as

$$
I_{\delta}(r)=E(r) E(r)^{*}=I_{0} \sin ^{2}[\delta(r) / 2] .
$$

Here, $I_{0} \equiv 1$ is the intensity of a laser beam that probes blood plasma. It is clear that the value of phase shift $\delta(r)$ can be determined using a direct measurement of the intensity $I_{\delta}(r)$ in the given point $(r)$ of the laser image

$\delta(r)=2 \arcsin \sqrt{I_{\delta}(r)}$.

Using the relations (10) to (14), one can obtain the expression for the algorithm providing determination of the CDC module describing the laser image of blood plasma in the points $r_{1}$ and $r_{2}$

$$
\left|\mu\left(r_{1}, r_{2}\right)\right|=0.5\left(1+\cos 2\left(\arccos \sqrt{I\left(r_{1}\right)}-\arccos \sqrt{I\left(r_{2}\right)}\right)\right)^{-1} .
$$

\section{Method to measure the coordinate distribution of the CDC module over the points of a laser image}

Shown in Fig. 1 is the optical scheme to measure coordinate distribution of the CDC module for laser images of blood plasma $[13,32]$.

Illumination of blood plasma layers (smears) was performed using a parallel beam $\left(\varnothing=10^{4} \mu \mathrm{m}\right)$ from a He-Ne laser $1(\lambda=0.6328 \mu \mathrm{m})$. The transmission plane of the polarizer 4 and the axis of the maximum velocity in the quarter-wave plate 5 made the angle $\Theta=45^{\circ}$. The image of blood plasma samples 6 was projected using the micro-objective 7 into the plane of a lightsensitive area $(r \equiv m \times n=800$ pix $\times 600$ pix $)$ of CCD camera 10.

The transmission plane of the analyzer 9 was oriented at the angle $\Theta=-45^{\circ}$ relatively to the axis of the maximum velocity in the quarter-wave plate 8 , which provided formation of conditions for phase filtration (relations (10) to (14)) for the laser image of a blood plasma sample.

The CCD camera 10 provided measurements of discrete two-dimensional $(m \times n)$ distributions for the intensity $I_{\delta}(m \times n)$. Then, calculated in accord with

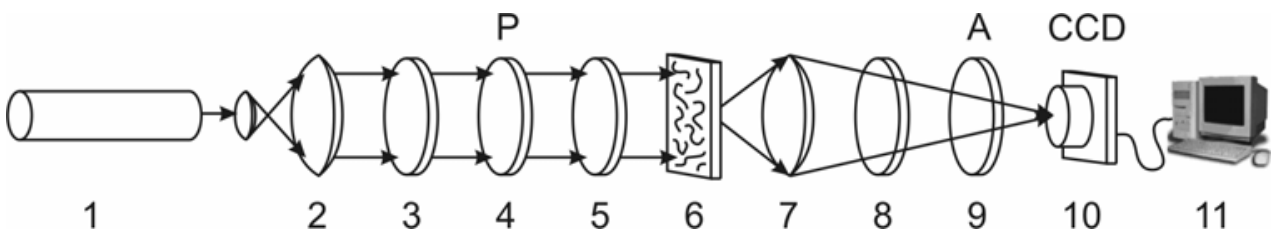

Fig. 1. Optical scheme for measurements. 1 - He-Ne laser; 2 - collimator; 3, 5, 8 - quarter-wave plates; 4, 9 - polarizer and analyzer, respectively; 6 - object; 7 - micro-objective (x4); 10 - CCD camera; 11 - personal computer. 
(14) were coordinate distributions $\delta(m \times n)$ scanned with the step $\Delta r=1 p i x$ along the lines $\left(\begin{array}{cccc}r_{11} & r_{11}+\Delta r & \ldots & r_{1 m} \\ \downarrow & & & \downarrow \\ \rightarrow & \rightarrow & \rightarrow & \rightarrow \\ \downarrow & & & \downarrow \\ r_{n 1} & r_{n 1}+\Delta r & \ldots & r_{n m}\end{array}\right)$ $\operatorname{array} \delta\left(\begin{array}{l}r_{11}, \ldots r_{1 m} \\ \ldots \ldots \ldots . \\ r_{n 1}, \ldots r_{n m}\end{array}\right)$.

Using the relation (15), for every pair of the points $\left(r_{i k}, r_{i k}+\Delta r\right)$ in the polarization-filtered laser image of blood plasma, we determined the value of CDC module $\mu\left(r_{i k}, r_{i k}+\Delta r\right)$.

As a result, we obtained the coordinate distribution $\mu\left(\begin{array}{ccc}\left(r_{11} ; r_{11}+\Delta r\right) & \ldots & \left(r_{1 m-1}, r_{1 m-1}+\Delta r\right) \\ \ldots & \ldots & \ldots \\ \left(r_{n 1}, r_{n 1}+\Delta r\right) & \ldots & \left(r_{n m-1}, r_{n m-1}+\Delta r\right)\end{array}\right)$ that, in what follows, we shall name as the correlation-phase map (CPM) for the blood plasma image.

\section{Algorithms for the complex statistical, correlation and fractal analysis of CPM}

To objectively estimate the distributions $\mu(x=1 \div m-1, y=1 \div n)$ for blood plasma laser images, we used the complex statistical, correlation and fractal analysis of their coordinate structure. The set of statistical moments of the first to fourth orders $Z_{j=1,2,3,4}^{\mu}$ was calculated using the following relations $[14,15,34$, $35,39]$

$$
\begin{aligned}
& Z_{1}^{\mu}=\frac{1}{N} \sum_{i=1}^{N}\left|\mu_{i}\right|, Z_{2}^{\mu}=\sqrt{\frac{1}{N} \sum_{i=1}^{N} \mu_{i}^{2}}, \\
& Z_{3}^{\mu}=\frac{1}{\left(Z_{2}^{\mu}\right)^{3}} \frac{1}{N} \sum_{i=1}^{N} \mu_{i}^{3}, Z_{4}^{\mu}=\frac{1}{\left(Z_{2}^{\mu}\right)^{2}} \frac{1}{N} \sum_{i=1}^{N} \mu_{i}^{4} .
\end{aligned}
$$
camera.

Here, $N$ is the amount of pixels in the digital

The correlation analysis of CPM is based on the autocorrelation method with using the function $[27,30$, 35]

$$
K_{i=1 \div n}^{\mu}(\Delta m)=\lim _{m \rightarrow 0} \frac{1}{m} \int_{1}^{m}[\mu(m)][\mu(m-\Delta m)] d m .
$$

Here, $(\Delta m=1$ pix $)$ is the "step" for changing coordinates $(x=1 \div m)$ of CDC distribution for the separate $i$ - th line of pixels in the digital camera.
The net expression for the autocorrelation function was obtained using averaging the partial functions (expression (17)) over all the lines $i=1 \div n$

$K^{\mu}(\Delta m)=\frac{\sum_{i=1}^{n} K_{i}^{\mu}(\Delta m)}{n}$.

To quantitatively characterize the autocorrelation dependences $K^{\mu}(\Delta m)$, we chose:

- "correlation area" $S^{\mu}$

$S^{\mu}=\int_{1}^{m} K^{\mu}(\Delta m) d m$

- "correlation moment" $Q_{4}^{\mu}$ that defines the excess for Gramm-Charlie expansion

$Q=\frac{\sum_{i=1}^{N}(K(\Delta m))_{i}^{4}}{\left(\sum_{i=1}^{N}(K(\Delta m))_{i}^{2}\right)^{2}}$

The fractal analysis of $\mu(m \times n)$ distributions was based on calculations of logarithmic dependences $\log J(\mu)-\log d^{-1}$ for power spectra $J(\mu)$

$J(\mu)=\int_{-\infty}^{+\infty} \mu \cos 2 \pi v d v$,

where $v=d^{-1}$ are the spatial frequencies that are determined by geometrical sizes $(d)$ of structural elements in laser images inherent to blood plasma layer.

Using the least squares method, the dependences $\log J(\mu)-\log d^{-1}$ were approximated to the curves $V(\eta)$, the straight parts of which provided determination of the slope angles $\eta$ and fractal dimensions $F^{\mu}[34]$ corresponding to them

$F^{\mu}=3-\operatorname{tg} \eta$.

Classification of the coordinate distributions $\mu(m \times n)$ was performed in accord with the following criteria [13-15]:

- fractal or self-similar, when the slope is constant ( $\eta=$ const ) within the limits of 2 or 3 decades for changing sizes $d$;

- $\mu(m \times n)-$ multi-fractal, when there are several slope angles $V(\eta)$;

- $\mu(m \times n)$ - random, when any stable slope angles $V(\eta)$ are absent over the whole interval of changing the sizes $d$.

All the distributions $\log J(\mu)-\log d^{-1}$ were characterized with the dispersion 
$D^{\mu}=\sqrt{\frac{1}{N} \sum_{i=1}^{N}\left[\log J(\mu)-\log d^{-1}\right]_{i}^{2}}$

\section{Diagnostic efficiency of the PCM and CPM methods for laser images of human blood plasma of patients in different physiological state}

Summarized in this Chapter are the data of comparative investigation aimed at the structure of blood plasma by using the methods of polarization-correlation $[27,30,35$, 38 ] and correlation-phase [36] mapping its laser images.

We investigated blood plasma samples (Fig. 2) taken from two group of patients - healthy (group 1 $q=27$ ) and those with prostate cancer (group 2 $q=25)$.

Our technique of sample preparation involved uniform applying the blood plasma smear on optically homogeneous glass with the following drying it at room temperature for 24 hours. As a result, we obtained optically-thin (extinction coefficient $\tau \leq 0.1$ ) layers with the geometrical thickness of 7 to $10 \mu \mathrm{m}$.

Summarized in Fig. 3 is the series of coordinate distributions for the CDMP module (fragments (1), (2)) and CPM (fragments (3), (4)); histograms $H(V)$ (fragments (5), (6)) and $H(\mu)$ (fragments (7), (8)); autocorrelation functions $K^{V}(\Delta m)$ (fragments (9), (10)) and $K^{\mu}(\Delta m)$ (fragments (11), (12)), as well as logarithmic dependences $\log J^{V}-\log d^{-1}$ (fragments (13), (14)) and $\log J^{\mu}-\log d^{-1}$ (fragments (15), (16)) that characterize polarization-correlation $V(m \times n)$ and correlation-phase $\mu(m \times n)$ maps of laser images corresponding to blood plasma layers of a healthy patient (fragments (1), (3), (5), (7), (9), (11), (13), (15)) and that with prostate cancer (fragments (2), (4), (6), (8), (10), (12), (14), (16)).

Our comparative analysis of this set of experimental data on statistical, correlation and fractal structures of PCM (Fig. 3, fragments (1), (2), (5), (6), (9), (10), (13), (14)) for laser images of blood plasma in both groups (Fig. 2) shows:

1. The histograms $H(V)$ (fragments (5), (6)) of distributions for the CDMP value $V$ in the corresponding PCM (fragments (1), (2)) are dependences with the clearly pronounced extreme $(V=1)$. This fact is indicative of a high degree of polarization homogeneity in blood plasma laser images. From the physical viewpoint, it can be explained by weakly pronounced polarization modulation of laser radiation, which could be provided by protein polycrystalline networks in blood plasma. As follows from relations (1) and (2), at low values $\delta=0.07 \ldots 0.15 \mathrm{rad}$ the values of elements in the Jones matrix tend to their limiting meanings $d_{11,22} \rightarrow 1$, $d_{12,21} \rightarrow 0$. In other words, the coordinate distribution of polarization states in a blood plasma laser image is close to polarization of the probing beam.

2. Autocorrelation functions $K^{V}(\Delta m)$ of CDMP coordinate distributions $V(m \times n)$ decay in a smooth and monotonic manner (fragments (9), (10)), which is also indicative of polarization homogeneity in blood plasma laser images of both groups.

3. Correlation consistency of coordinate distributions $V(m \times n)$ is reflected in their fractal structure. As seen, approximating curves to logarithmic dependences $\log J^{V}-\log d^{-1}$ possess one stable slope angle (fragments (13), (14)) over the whole range of changing the geometrical sizes $d$.

Quantitatively, the PCM of laser images inherent to blood plasma of healthy patients and those with cancer have been illustrated with statistical $\left(Z_{i=1-4}^{V}\right)$, correlation $\left(S^{V}, Q^{V}\right)$ and fractal $\left(F^{V}, D^{V}\right)$ parameters (Table 1).

The comparative analysis of the obtained data set (Table 1) did not reveal any reliable criteria for differentiation of blood plasma laser images for both groups. As can be seen, the values and ranges for changing the statistical, correlation and fractal parameters that characterize coordinate CDMP distributions are "overlapped" (see fragments (5) and (6)).

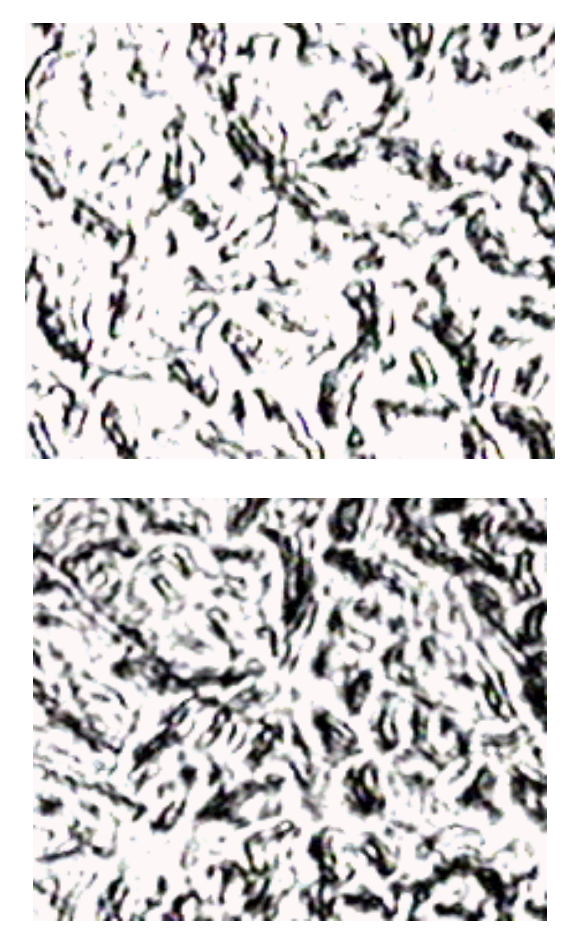

Fig. 2. Laser images $\left(\Theta=90^{0}\right)$ of protein polycrystalline networks inherent to blood plasma. Explanations are given in the text. 


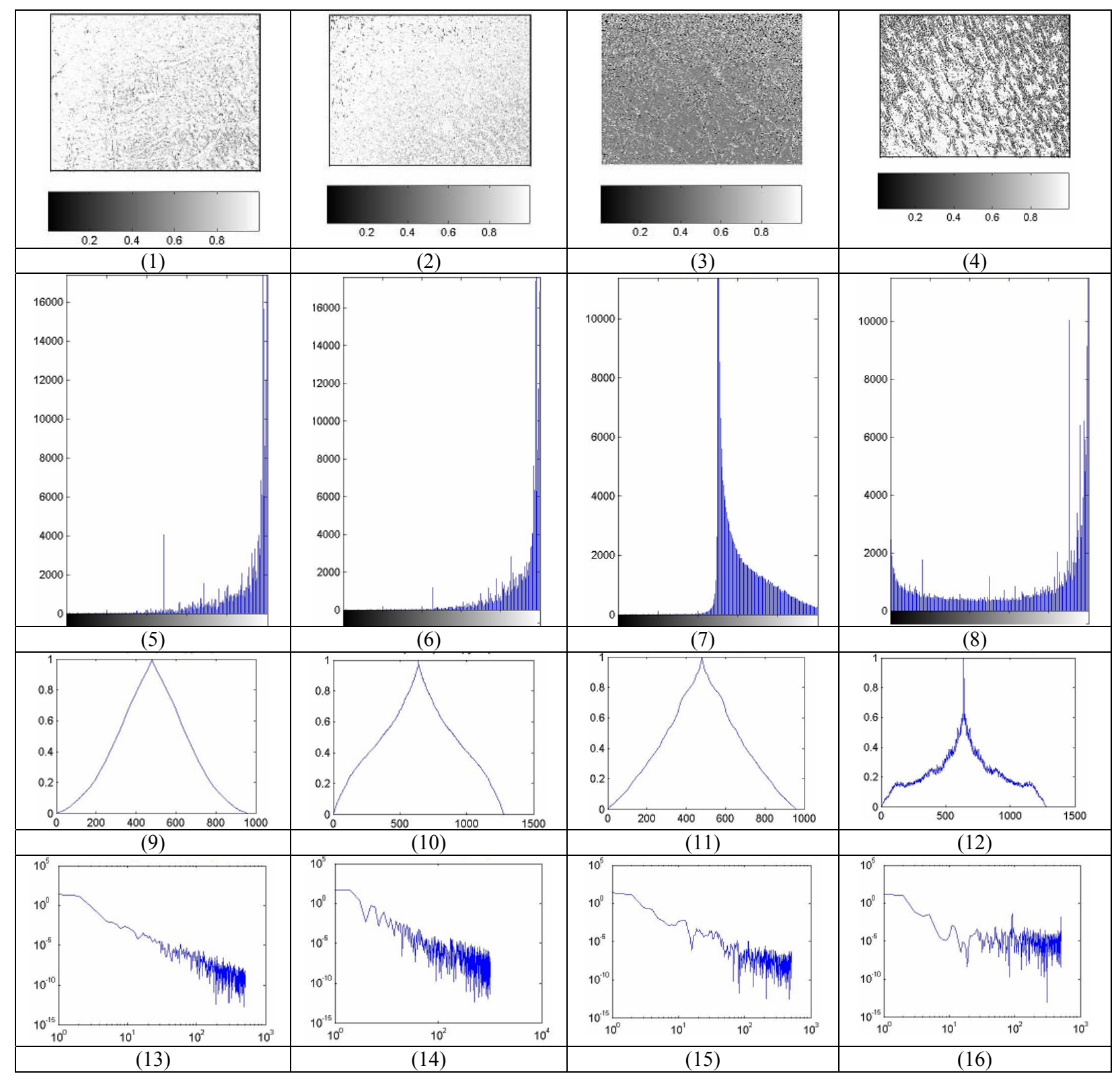

Fig. 3. Coordinate, statistical, correlation and fractal structures of PCM and CPM of human blood plasma for patients in different physiological states. Explanations are given in the text.

As to its diagnostic performance, the method of correlation-phase mapping is more sensitive to changes in the structure of polycrystalline network in human blood plasma taken from the patient with prostate cancer. Statistically, these differences can be observed in transformation of CDC distributions (Fig. 3, fragments (3) and (4)) of corresponding laser images.

The histograms $H(\mu)$ are characterized with availability of local extremes in a wider range of changes in this correlation-phase parameter (fragments (7), (8)). This fact indicates growth in phase modulation, which is typical for blood plasma images in the group 2 . The analysis of Exp. (15) shows that, even for low values of phase shifts $\delta=0.07 \ldots 0.15 \mathrm{rad}$, the CDC value undergoes significant changes within the range $0.65 \leq \mu \leq 0.98$. Autocorrelation functions $K^{\mu}(\Delta m)$ of coordinate distributions $\mu(m \times n)$ sharply drop (fragment (12)), which is also indicative of phase inhomogeneity in laser images describing blood plasma of patients with cancer.

The most pronounced is the change in coordinate structure of $\mu(m \times n)$ distributions, which is caused by pathological changes and is reflected in its transformation from a fractal type (fragment (15)) to the random one (fragment (16)). 
Table 1. Statistical ( $\left.Z_{i=1-4}^{V}\right)$, correlation $\left(S^{V}, Q^{V}\right)$ and fractal $\left(F^{V}, D^{V}\right)$ parameters of polarization-correlation maps for laser images of blood plasma

\begin{tabular}{|c|c|c|c|c|c|c|c|c|}
\hline Parameters & $Z_{1}^{V}$ & $Z_{2}^{V}$ & $Z_{3}^{V}$ & $Z_{4}^{V}$ & $S^{V}$ & $Q^{V}$ & $F^{V}$ & $D^{V}$ \\
\hline $\begin{array}{l}\text { Group } 1 \\
\text { (27 samples) }\end{array}$ & $\begin{array}{c}0.97 \\
\pm \\
0.02\end{array}$ & $\begin{array}{c}0.05 \\
\pm \\
0.007\end{array}$ & $\begin{array}{c}0.08 \\
\pm \\
0.009\end{array}$ & $\begin{array}{c}0,73 \\
\pm \\
0.87\end{array}$ & $\begin{array}{c}0.26 \\
\pm \\
0.011\end{array}$ & $\begin{array}{c}0.21 \\
\pm \\
0.032\end{array}$ & $\begin{array}{c}2.42 \\
\pm \\
0.011\end{array}$ & $\begin{array}{c}0.18 \\
\pm \\
0.021\end{array}$ \\
\hline $\begin{array}{l}\text { Group } 2 \\
\text { (25 samples) }\end{array}$ & $\begin{array}{c}0.96 \\
\pm \\
0.01\end{array}$ & $\begin{array}{c}0.06 \\
\pm \\
0.008\end{array}$ & $\begin{array}{c}0.1 \\
\pm \\
0.017\end{array}$ & $\begin{array}{c}0.67 \\
\pm \\
0.075\end{array}$ & $\begin{array}{c}0.24 \\
\pm \\
0.013\end{array}$ & $\begin{array}{c}0.27 \\
\pm \\
0.036\end{array}$ & $\begin{array}{c}2.49 \\
\pm \\
0.012\end{array}$ & $\begin{array}{c}0.23 \\
\pm \\
0.027\end{array}$ \\
\hline
\end{tabular}

Table 2. Statistical $\left(Z_{i=1-4}^{\mu}\right)$, correlation $\left(S^{V}, Q^{V}\right)$ and fractal $\left(F^{V}, D^{V}\right)$ parameters of correlation-phase maps for blood plasma laser images

\begin{tabular}{|l|c|c|c|c|c|c|c|c|}
\hline Parameters & $Z_{1}^{\mu}$ & $Z_{2}^{\mu}$ & $Z_{3}^{\mu}$ & $Z_{4}^{\mu}$ & $S^{\mu}$ & $Q^{\mu}$ & $F^{\mu}$ & $D^{\mu}$ \\
\hline Group 1 & 0.91 & 0.07 & 0.09 & 0.27 & 0.24 & 0.23 & 1.952 & 0.21 \\
(27 samples) & \pm & \pm & \pm & \pm & \pm & \pm & \pm & \pm \\
& 0.009 & 0.009 & 0.011 & 0.031 & 0.01 & 0.032 & 0.014 & 0.026 \\
\hline Group 2 & 0.79 & 0.15 & 0.325 & 1.39 & 0.17 & 0.86 & & 0.28 \\
(25 samples) & \pm & \pm & \pm & \pm & \pm & \pm & - & \pm \\
& 0.08 & 0.018 & 0.017 & 0,017 & 0.012 & 0.091 & & 0.036 \\
\hline
\end{tabular}

From the physical viewpoint, these changes in CPM of blood plasma laser images for the group 2 can be related with growth of birefringence in polycrystalline networks due to increasing the concentration of albumin and globulin proteins. The mentioned biochemical process causes growth of the phase modulation $\delta(m \times n)$ of laser radiation. Statistically, this effect manifests itself as spreading the range of changes in the value of CDC module as well as in lowering the correlation consistency of various CPM points and formation of the random distribution $\mu(m \times n)$. Quantitatively, CPM of laser images corresponding to blood plasma of patients both healthy and with cancer are illustrated using the statistical $\left(Z_{i=1-4}^{\mu}\right)$, correlation $\left(S^{V}, Q^{V}\right)$ and fractal $\left(F^{V}, D^{V}\right)$ parameters (Table 2).

\section{Conclusions}

The obtained results of studying the statistical, correlation and fractal structures of correlation-phase maps corresponding to blood plasma laser images for both groups enabled us to formulate the following objective criteria for their differentiation:

1. The values of statistical moments of the third and fourth orders, which characterize $\mu(m \times n)$ distributions of laser images for the group 2 samples, are 3.45 and 4.17 times higher than the analogous parameters $Z_{3}^{\mu}, Z_{4}^{\mu}$ of blood plasma in the group 1 .

2. The values of correlation area $S^{\mu}$ and correlation moments $Q^{\mu}$ differ 1.4 and 3.6 times, respectively, for both groups of blood plasma.
3. The fractal $\mu(m \times n)$ distribution of laser images for samples of blood plasma in the group 1 is transformed into the random one for the samples from the group 2.

Thus, we have determined the set of objective criteria, using which one can differentiate coordinate distributions of the module of complex degree of coherency for laser images of human blood plasma. Also, we have demonstrated the diagnostic sensitivity of the offered method to pathologic states observed in patients with cancer.

\section{References}

1. M. Born and E. Wolf, Principles of Optics (Cambridge Univ. Press, Cambridge), (1999).

2. E. Wolf, "Unified theory of coherence and polarization of random electromagnetic beams," Phys. Lett. A. 312, 263-267, (2003).

3. E. Wolf, "Correlation-induced changes in the degree of polarization, the degree of coherence, and the spectrum of random electromagnetic beams on propagation," Opt. Lett. 28, 1078-1080, (2003).

4. J. M. Movilla, G. Piquero, R. Martínez-Herrero, P. M. Mejías, "Parametric characterization of nonuniformly polarized beams," Opt. Commun. 149, 230-234, (1998).

5. Claudia Mujat and Aristide Dogariu, "Statistics of partially coherent beams: a numerical analysis," J. Opt. Soc. Am. A 21, 1000-1003 (2004).

6. F.Gori, "Matrix treatment for partially polarized, partially coherent beams," Opt. Lett. 23, 241-243 (1998). 
7. E. Wolf, "Significance and measurability of the phase of a spatially coherent optical field," Opt. Lett. 28, 5-6 (2003).

8. Mircea Mujat and Aristide Dogariu, "Polarimetric and spectral changes in random electromagnetic fields," Opt. Lett. 28, 2153-2155 (2003).

9. J. Ellis, A. Dogariu, S. Ponomarenko, E. Wolf, "Interferometric measurement of the degree of polarization and control of the contrast of intensity fluctuations," Opt. Lett. 29, 1536 - 1538 (2004).

10. M. Mujat, A. Dogariu, G. S. Agarwal, "Correlation matrix of a completely polarized, statistically stationary electromagnetic field," Opt. Lett., 29, 1539 -1541 (2004).

11. Adela Apostol and Aristide Dogariu, "First- and second-order statistics of optical near fields," Opt. Lett. 29, 235-237 (2004).

12. J.Ellis and A.Dogariu, "Complex degree of mutual polarization,” Opt.Lett., 29, 536-538 (2004).

13. O. V. Angelsky, A. G. Ushenko, Yu. A. Ushenko, V. P. Pishak, "Statistical and Fractal Structure of Biological Tissue Mueller Matrix Images”, in Optical Correlation Techniques and Applications, Oleg V. Angelsky, ed. (Washington: Society of Photo-Optical Instrumentation Engineers), pp. 213 266 (2007).

14. O.V. Angelsky, A.G. Ushenko, Yu.A. Ushenko, V.P. Pishak, and A.P. Peresunko, "Statistical, Correlation, and Topological Approaches in Diagnostics of the Structure and Physiological State of Birefringent Biological Tissues”, in Handbook of Photonics for Biomedical Science, Valery V. Tuchin, ed. (USA: CRC Press), pp. 2167 (2010).

15. Alexander G. Ushenko and Vasilii P. Pishak, "Laser Polarimetry of Biological Tissue: Principles and Applications", in Handbook of Coherent-Domain Optical Methods: Biomedical Diagnostics, Environmental and Material Science, Valery V. Tuchin, ed. (Boston: Kluwer Academic Publishers), pp. 93-138 (2004).

16. A.G. Ushenko, S.B. Ermolenko, D.N. Burkovets, Yu.A. Ushenko, "Polarization Microstructure of Laser Radiation Scattered by Optically Active Biotissues," Optics and Spectroscopy 87(3), 434439 (1999).

17. A.G. Ushenko, "Laser diagnostics of biofractals," Quantum Electron. 29(12), 1078-1084 (1999).

18. O.V. Angel'skii, A.G. Ushenko, A.D. Arkhelyuk, S.B. Ermolenko, D.N. Burkovets, "Structure of matrices for the transformation of laser radiation by biofractals," Quantum Electron. 29(12), 1074-1077 (1999).

19. O.V. Angel'skii, A.G. Ushenko, A.D. Arheluk, S.B. Ermolenko, D.N. Burkovets, "Scattering of Laser Radiation by Multifractal Biological Structures," Optics and Spectroscopy 88(3), 444-448 (2000).
20. A.G. Ushenko, "Polarization Structure of Biospeckles and the Depolarization of Laser Radiation," Optics and Spectroscopy 89(4), 597601 (2000).

21. O.V. Angel'skii, A.G. Ushenko, S.B. Ermolenko, D.N. Burkovets, V.P. Pishak, Yu.A. Ushenko, O.V. Pishak, "Polarization-Based Visualization of Multifractal Structures for the Diagnostics of Pathological Changes in Biological Tissues," Optics and Spectroscopy 89(5), 799-805 (2000).

22. O.V. Angel'skii, A.G. Ushenko, A.D. Arheluk, S.B. Ermolenko, D.N. Burkovets, Yu.A. Ushenko, "Laser Polarimetry of Pathological Changes in Biotissues," Optics and Spectroscopy 89(6), 973979 (2000).

23. A.G. Ushenko, "The Vector Structure of Laser Biospeckle Fields and Polarization Diagnostics of Collagen Skin Structures," Laser Physics 10(5), 1143-1149 (2000).

24. A.G. Ushenko, S.B. Ermolenko, D.N. Burkovets, Yu.A. Ushenko, "Laser polarimetry of the orientation structure of bone tissue osteons," Journal of Applied Spectroscopy 67(1), 65-69 (2000).

25. O.V. Angel'skii, A.G. Ushenko, A.D. Arheluk, S.B. Ermolenko, D.N. Burkovets, Yu.A. Ushenko, "Polarization-Phase Visualization and Processing of Coherent Images of Fractal Structures of Biotissues," Journal of Applied Spectroscopy 67(5), 919-923 (2000).

26. A.G. Ushenko, D.N. Burkovets, and Yu.A. Ushenko, "Laser polarization visualization and selection of biotissue images," Laser Physics 11(5), 624-631 (2001).

27. O.V. Angel'skii, A.G. Ushenko, D.N. Burkovets, Yu.A. Ushenko, "Polarization-correlation analysis of anisotropic structures in bone tissue for the diagnostics of pathological changes," Optics and Spectroscopy 90(3), 458-462 (2001).

28. A.G. Ushenko, "Laser polarimetry of polarizationphase statistical moments of the object field of optically anisotropic scattering layers," Optics and Spectroscopy 91(2), 313-316 (2001).

29. A.G. Ushenko, "Polarization contrast enhancement of images of biological tissues under the conditions of multiple scattering," Optics and Spectroscopy 91(6), 937-940 (2001).

30. A.G. Ushenko, "Laser probing of biological tissues and the polarization selection of their images," Optics and Spectroscopy 91(6), 932-936 (2001).

31. A.G. Ushenko, "Correlation processing and wavelet analysis of polarization images of biological tissues," Optics and Spectroscopy 91(5), 773-778 (2002).

32. A.G. Ushenko, "Polarization correlometry of angular structure in the microrelief pattern or rough surfaces," Optics and spectroscopy 92(2), 227-229 (2002). 
33. A.G. Ushenko, D.N. Burkovets, Yu.A. Ushenko, "Polarization-Phase Mapping and Reconstruction of Biological Tissue Architectonics during Diagnosis of Pathological Lesions," Optics and Spectroscopy 93(3), 449-456 (2002).

34. Oleg V. Angelsky, Alexander G. Ushenko, Dimitry N. Burkovets, Yuriy A. Ushenko, "Laser polarization visualization and selection of biotissue images," Optica Applicata 32(4), 591-602 (2002).

35. O.V. Angelsky, A.G. Ushenko, Yu.A. Ushenko, "Polarization reconstruction of orientation structure of biological tissues birefringent architectonic nets by using their Mueller-matrix speckle-images," Journal of Holography and Speckle 2(2), 72-79 (2002).

36. E.I. Olar, A.G. Ushenko, and Yu.A. Ushenko, "Correlation Microstructure of the Jones Matrices for Multifractal Networks of Biotissues," Laser Physics 14, 1012-1018 (2004).
37. O.V. Angelsky, A.G. Ushenko, Yu.A. Ushenko, A.O. Angelskaya, "Fractal structure of 2D Mueller matrix images of biotissues," Ukrainian Journal of Physical Optics 6(1), 13-23 (2004).

38. Oleg V. Angelsky, G.V. Demianovsky, A.G. Ushenko, D.N. Burkovets, and Yu.A. Ushenko, "Wavelet analysis of two-dimensional birefringence images of architectonics in biotissues for diagnosing pathological changes," J. Biomed. Opt. 9, 679-690 (2004).

39. Oleg V. Angelsky, Alexander G. Ushenko, Dimitry N. Burcovets, Yuriy A. Ushenko, "Polarization visualization and selection of biotissue image twolayer scattering medium," J. Biomed. Opt. 10(1), 014010 (2005). 